\title{
Particulates from hydrophilic-coated guiding sheaths embolise to the brain
}

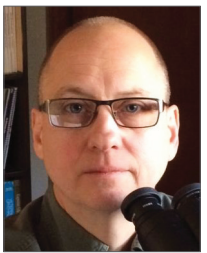

James R.L. Stanley ${ }^{1}$, DVM, MS; Abraham R. Tzafriri*, PhD; Kathryn Regan ${ }^{1}$, HT(ASCP)CM; Alan LaRochelle ${ }^{1}$, BS; Gee Wong ${ }^{1}$, BS, HT; Brett G. Zani ${ }^{1}$, PhD; Peter M. Markham¹ ${ }^{1}$ MS; Lynn Bailey ${ }^{1}$, AS, LATG; Anna Spognardi¹, BA; Gregory A. Kopia², PhD; Elazer R. Edelman ${ }^{3}, \mathrm{MD}, \mathrm{PhD}$

1. CBSET, Inc., Lexington, MA, USA; 2. Kopia Consulting, Hillsborough, NJ, USA; 3. Institute for Medical and Engineering Science, Massachusetts Institute of Technology, Cambridge, MA, USA, and Department of Medicine, Brigham and Women's Hospital and Harvard Medical School, Boston, MA, USA

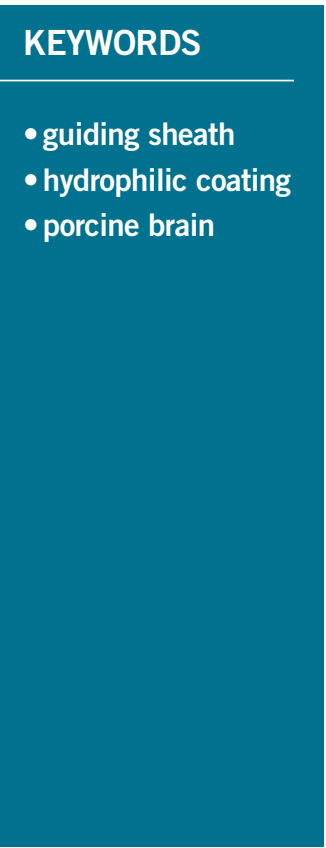

\section{Abstract}

Aims: We sought to evaluate the incidence of embolic material in porcine brains following vascular interventions using hydrophilic-coated sheaths.

Methods and results: A new self-expanding stent and delivery system (SDS) was deployed through a hydrophilic-coated (Flexor ${ }^{\circledR}$ Ansel; Cook Medical, Bloomington, IN, USA) guiding sheath into the iliac and/or carotid arteries of 23 anaesthetised Yucatan mini swine. The animals were euthanised at three, 30, 90 and 180 days and their brains were removed for histological analysis. In an additional single control animal, the guiding sheath was advanced but no SDS was deployed. Advancement of the coated guiding sheath with or without the SDS was associated with frequent foreign material in the arterioles of the brain. The embolic material was amorphous, non-refractile, non-crystalline, non-birefringent and typically lightly basophilic with a slightly stippled appearance on haematoxylin and eosin (H\&E) stain. Material was observed at all time points involving $54 \%$ of all study animals (i.e., test and control) and in vitro after incubation in $0.9 \%$ saline.

Conclusions: The hydrophilic coating on a clinically used guiding sheath readily avulses and embolises to the brain during deployment in a porcine model. Further documentation of this effect and monitoring in clinical scenarios are warranted.

*Corresponding author: CBSET Inc., 500 Shire Way, Lexington, MA, 02421, USA.

E-mail: rtzafriri@cbset.org 


\section{Introduction}

Medical catheters are used ubiquitously in diagnostic and therapeutic interventional procedures in large numbers of patients each year. These catheters permit external access to deep body spaces for monitoring, imaging, sampling and delivery of therapeutic drugs such as local thrombolysis, and/or deployment of medical devices, such as endovascular stents, aneurysm coils, ablation catheters, and transcatheter valves. Such catheter-based procedures are generally far less traumatic alternatives to surgical means.

Yet, percutaneous catheter insertion is not without risk. Patients undergoing percutaneous interventional procedures are extensively anticoagulated, raising the spectre of imbalance between haemorrhage (bleeding) and thrombosis (clotting). As larger catheters are employed for increasingly complex interventions in the periphery and across or within valves and ventricles, the risk of vascular injury rises. Guiding sheaths which have been modified by the addition of a hydrophilic coating on their exterior enhance catheter delivery and advancement. Though in clinical use for more than a decade, there have been reports of complications with these hydrophiliccoated devices. The majority of reported cases of adverse events with hydrophilic-coated catheters involve local inflammation following radial artery catheter insertion ${ }^{1,2}$. Such adverse events have been histologically correlated with intralesional foreign material that appeared filamentous, non-birefringent and blue-grey with haematoxylin and eosin (H\&E) staining, and similar in appearance to hydrophilic coating scraped from the exterior surface of involved devices ${ }^{1}$.

However, foreign material adverse clinical events have not been limited to the radial artery. Indeed, non-refractile, non-polarisable, granular basophilic foreign material was found in either biopsy or autopsy samples of tissues including lung, foot and brain ${ }^{3,4}$. Another more recent report has described the finding of foreign material following histopathological analyses of brain sections from three patients in whom fatal ipsilateral intracerebral haemorrhages developed several days after uneventful pipeline embolisation device treatment of supraclinoid aneurysms. $s^{5}$. In each case, a basophilic intra-arterial filamentous foreign material was observed in brain autopsy histology sections that not only resembled shavings of the coatings shed during benchtop manipulation of the hydrophiliccoated guiding sheath, but were spectroscopically identical and identified as polyvinylpyrrolidone (PVP) $)^{5}$.

As the above studies were conducted in patients with some underlying vascular pathology with numerous and different interventional devices, additional instructive data could be gained via the use of hydrophilic material-coated catheters in experimental animals where both the access devices and the test devices are consistent among the study population. Here, we report on a hydrophilic-coated guiding sheath that was associated with foreign material in brain sections of treated animals and material shedding in vitro. To our knowledge this is the first such preclinical report of foreign material findings in animal brains and of the correlation of animal findings with the propensity for in vitro shedding.

\section{Methods}

Studies were conducted at CBSET, Inc. (Lexington, MA, USA) and adhered to the Guide for the Care and Use of Laboratory Animals ${ }^{6}$ under an approved Institutional Animal Care and Use Committee (IACUC) protocol, in compliance with the Animal Welfare Act and the Food and Drug Administration Regulations and their amendments.

\section{IN VIVO}

Twenty-four adult, healthy Yucatan mini swine weighing 39.4-45.5 kg were pre-treated with $650 \mathrm{mg}$ aspirin and $300 \mathrm{mg}$ clopidogrel orally to reduce the potential for thrombosis, and thereafter $81 \mathrm{mg}$ aspirin and $75 \mathrm{mg}$ clopidogrel orally each day until euthanised. On the day of surgery, animals were sedated with Telazol (4-6 mg/ $\mathrm{kg}$, i.m.) and anaesthetised with isofluorane in $100 \%$ oxygen. Following intubation and pre-treatment with nifedipine (10 mg sublingually), a 90 $\mathrm{cm} 6$ Fr guiding sheath (Flexor ${ }^{\circledR}$ Ansel Guiding Sheath with Check$\mathrm{Flo}^{\circledR}$ Hemostasis Valve; Cook Medical, Bloomington, IN, USA) with a hydrophilic coating ( $\mathrm{AQ}^{\circledR}$ hydrophilic coating) was placed in either the right common carotid artery (for iliac stent deployment) or the right femoral artery (for carotid artery deployment). Animals were pre-treated with heparin $(50-200 \mathrm{U} / \mathrm{kg})$ to achieve a target activated clotting time of approximately 200-275 seconds. Under fluoroscopic guidance, a guiding catheter was advanced through the guiding sheath over a guidewire to either the carotid or the iliac arteries. Stent and delivery systems (SDS) compatible with a 6 Fr introducer sheath were then advanced through the guiding sheaths to a predetermined arterial site and deployed. Two stents were overlapped in the carotid arteries but were deployed singly in the iliac arteries. Stent dimensions $(6 \times 60 \mathrm{~mm}$ or $7 \times 60 \mathrm{~mm})$ were selected for use based on vessel size. Nitroglycerine (50-200 $\mu \mathrm{g})$ was administered intra-arterially prior to obtaining angiograms. Following the interventional procedure, the accessed vessel was either ligated or repaired and the surgical incision closed in layers with suture. Animals were observed until recovered and monitored until full recovery from anaesthesia. Total dwell time for the guiding sheath ranged from 20 to 25 minutes. Animals were then euthanised post stenting at three days $(\mathrm{n}=4), 30$ days $(\mathrm{n}=7)$, 90 days $(n=8)$ or 180 days $(n=4)$. In one animal, the preliminary angiographic assessment indicated that the target vessels were inadequate to meet the size criteria for the intended stents. Since this animal received an identical guiding sheath but no stent deployment, it was maintained on study for 30 days to serve as an unstented control.

At the respective study endpoints, all animals underwent a complete physical examination by the institutional veterinarian (including evaluation of neuromuscular systems) followed by angiography, euthanasia, and necropsy. Stented vessels were pressure perfused $(100 \mathrm{mmHg}$ ) with $10 \%$ neutral buffered formalin (NBF) and removed. The entire brain was collected and placed in $10 \%$ NBF for histopathologic processing and assessment.

\section{IN VITRO}

Tip segments of a 9 Fr guiding sheath with a hydrophilic coating (Flexor Ansel AQ ${ }^{\circledR}$ hydrophilic coating [product code G49102]; Cook Medical) were placed in $0.9 \%$ saline and serially photographed 
over 60 minutes. Material was then scraped from the surfaces of the catheter segment and placed into the lumen of a swine carcass aorta (to retain the material during paraffin histologic processing), formalin fixed, embedded, sectioned, stained with $H \& E$ and examined microscopically.

\section{Histopathology}

Brains were fixed in NBF for at least seven days, after which they were embedded in paraffin. Six sections were obtained from each brain of the test animals and 28 sections obtained from the single control animal. Sections were mounted on glass slides, stained with $\mathrm{H} \& \mathrm{E}$ and examined microscopically by a board-certified veterinary pathologist using an Olympus BX41 microscope (Olympus Optical Corporation, Ltd., Tokyo, Japan).

\section{Statistical analysis}

Experimental values are reported descriptively as mean \pm standard deviation. Categorical data based on intravascular access point (carotid vs. femoral) and the evidence of brain emboli ("yes" when present, "no" when not) were pooled from all examined brain sections and statistically compared using a Fisher's exact test (SigmaPlot 11.0).

\section{Results}

\section{BRAIN HISTOPATHOLOGY}

Of the 24 animals treated with the hydrophilic-coated guiding sheath, the brains of 13 (54\%) exhibited evidence of an acellular intra-arterial foreign material (Figure 1), which, collectively, involved all major regions of the brain (i.e., forebrain, midbrain, hindbrain, brain stem and cerebellum). With H\&E stain, the material was amorphous

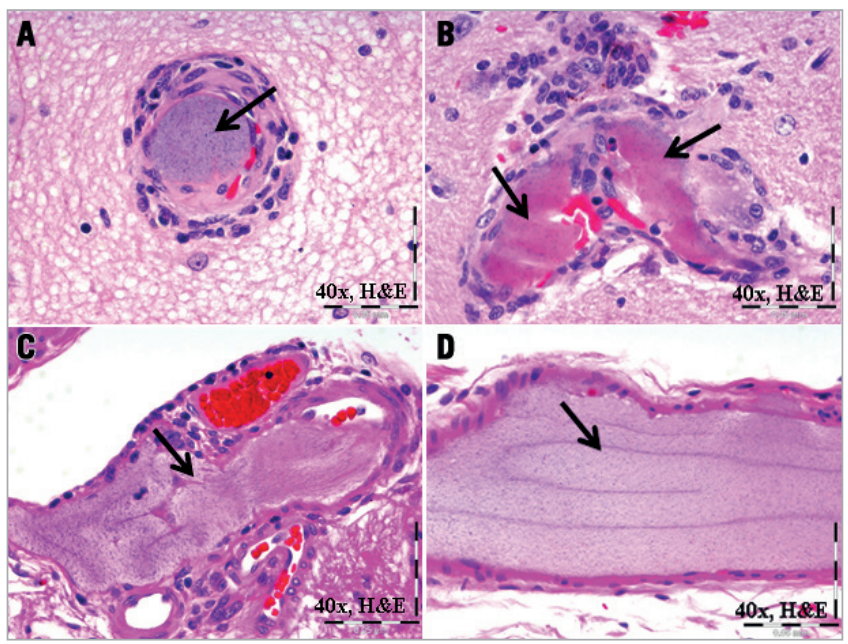

Figure 1. Intra-arterial foreign material (arrows) in porcine brain sections. Example of intra-arterial amorphous, stippled, basophilic (A) to eosinophilic $(B, C, D)$ foreign material with associated peri-arterial inflammatory cells in sections of 30-day porcine right midbrain (A, B), and 180-day forebrain (C) and cerebellum (D) following deployment of the hydrophilic-coated guiding sheath followed by distal peripheral stenting. (i.e., smeared and conforming), non-refractile, non-crystalline, nonbirefringent and typically lightly basophilic, and occasionally eosinophilic, with a slight stippled appearance. Frequently associated with the foreign material was the presence of variable chronic inflammatory infiltrates (i.e., lymphohistiocytic and/or multinucleated giant cells $[\mathrm{MNGC}]$ ), regardless of time point, which were minimal in magnitude and typically occurred around the arterioles containing the foreign material, and less frequently observed in the pia mater, choroid plexus, and/or neuropil. There was no evidence of acute inflammation (e.g., neutrophils, suppuration) and no time-related trends. Evidence of any significant adverse effects (e.g., infarction) was limited to a focus of resolving malacia in the brain of one treated animal (Figure 2). Similar to affected test animals, intra-arterial foreign material, and/or associated minimal lymphohistiocytic and MNGC, was also observed in the control animal which underwent preliminary angiography with access using the hydrophilic-coated guiding sheath but did not receive any stents (Figure 3).

\section{INCIDENCE AND BRAIN DISTRIBUTION}

Of the 24 animals exposed to the guiding sheath, arteriolar foreign material was observed in brain sections from one of five (20\%) animals accessed via the right femoral artery, while 12 of $19(63 \%)$ animals accessed via the right common carotid artery demonstrated brain foreign material. However, the trend for higher incidence of brain emboli with carotid versus femoral access was not statistically significant ( $\mathrm{p}=0.122$, Fisher's exact test).

Foreign material was observed in the brains of animals from all time points (Table 1). Fifty-four percent of all study animals (i.e.,

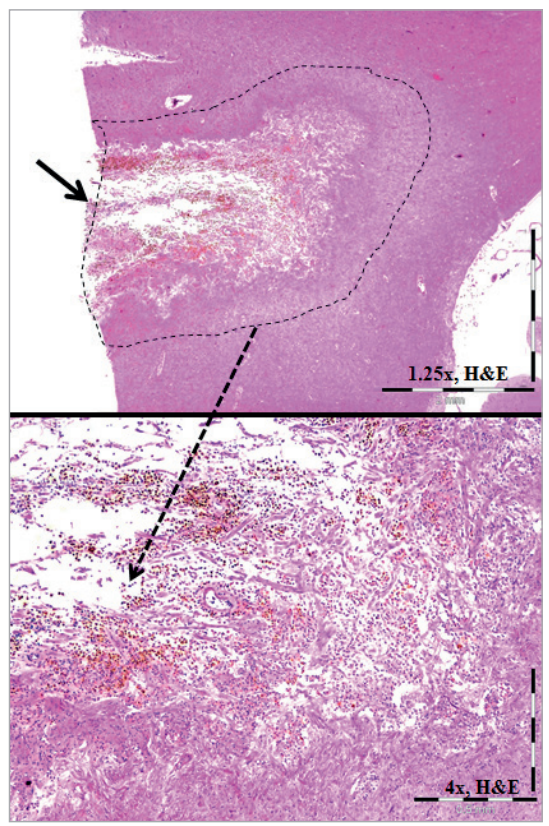

Figure 2. Example of local infarction. Note the focally extensive area of infarction illustrated by chronic, resolving malacia (within dashed lines, $1.25 \times$ image) characterised by fibrous astrocytosis and microglial haemosiderosis (4×image). 
Table 1. Incidence of foreign material observed in porcine brains from animals treated with the Cook guiding sheath.

\begin{tabular}{|c|c|c|c|c|c|c|c|}
\hline \multirow[b]{2}{*}{ Group } & \multirow[b]{2}{*}{ Time point } & \multicolumn{3}{|c|}{ Animal incidence } & \multicolumn{3}{|c|}{ Planar incidence } \\
\hline & & Total animals & $\begin{array}{l}\text { Animals with } \\
\text { foreign material }\end{array}$ & $\%$ & Total planes & $\begin{array}{c}\text { Planes with } \\
\text { foreign material }\end{array}$ & $\%$ \\
\hline \multicolumn{8}{|c|}{ Cook Medical guiding sheath } \\
\hline Control & Day 30 & 1 & 1 & $100 \%$ & 28 & 9 & $32 \%$ \\
\hline \multirow[t]{4}{*}{ Test } & Day 3 & 4 & 1 & $25 \%$ & 24 & 2 & $8 \%$ \\
\hline & Day 30 & 7 & 4 & $57 \%$ & 42 & 4 & $10 \%$ \\
\hline & Day 90 & 8 & 4 & $50 \%$ & 48 & 6 & $13 \%$ \\
\hline & Day 180 & 4 & 3 & $75 \%$ & 24 & 3 & $13 \%$ \\
\hline \multicolumn{2}{|c|}{ Total test animals } & 23 & 12 & $52 \%$ & 138 & 15 & $11 \%$ \\
\hline \multicolumn{2}{|l|}{ All animals } & 24 & 13 & $54 \%$ & 166 & 24 & $14 \%$ \\
\hline
\end{tabular}

all test and one non-stented control) had at least one fragment of foreign material observed in each brain section. Of the 138 histologic sections of brain tissue examined from all stent and delivery system (SDS) animals, representing major regions of the brain $(\mathrm{n}=6$ per animal), 15 (11\%) contained fragments of foreign material. By contrast, of the 28 histologic sections of brain tissue examined from the control animal (exposed only to a hydrophilic-coated sheath), nine (32\%) contained foreign material. When all animals are combined across treatments and time points $(n=24)$, the greatest incidence of foreign material was observed in the cerebellum with a lesser incidence in the forebrain, right midbrain, hindbrain and brain stem, with the least incidence observed in the left midbrain (Figure 4).

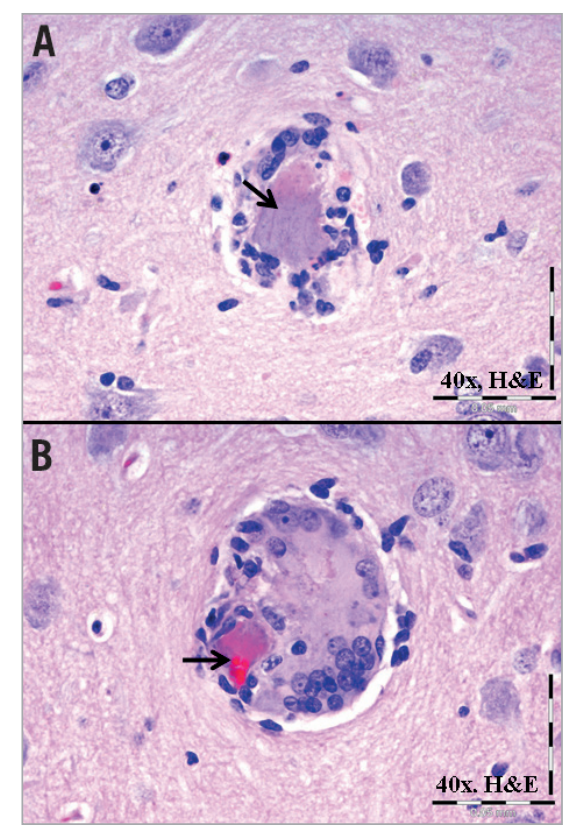

Figure 3. Foreign material in a brain section from the control animal. Intra-arterial accumulation of amorphous, stippled basophilic to eosinophilic material (arrows) from a non-stented control animal which had received only a hydrophilic-coated guiding sheath 30 days previously. Note the associated lymphohistiocytic infiltrates (A) and additional multinucleated giant cell infiltrates (B).

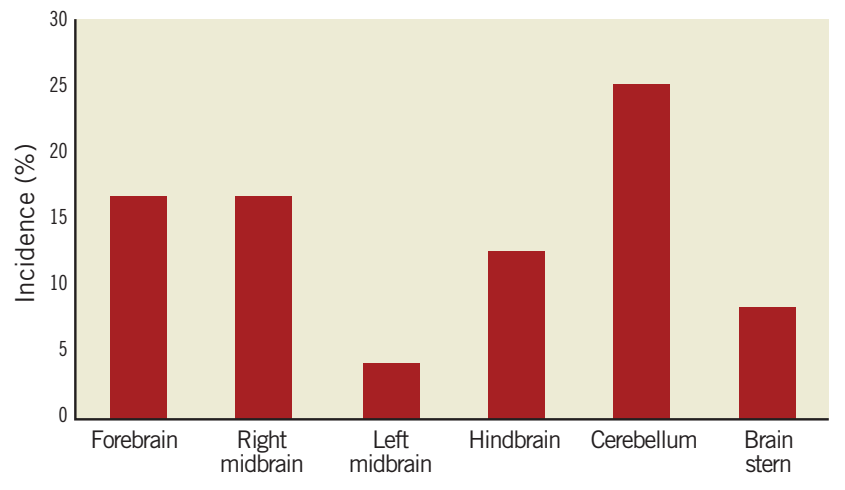

Figure 4. Distribution of hydrophilic coating material. Regional distribution of foreign material in porcine brains following use of guiding sheath. Incidence represents number of animals with material observed in the specified region divided by all animals $(n=24)$.

\section{IN VITRO HISTOPATHOLOGY}

For better assessment of the nature of the foreign material, a distal portion of a hydrophilic-coated guiding sheath was placed in $0.9 \%$ saline for 60 minutes. Avulsion of the hydrophilic coating from the sheath was noted when incubated in saline for as little as 15 minutes. Over time, the material (presumed hydrophilic coating) was observed to have increasingly separated/shed from the surface of the catheter. When samples of this material were scraped off the 60-minute incubation sheath sample (Figure 5) and placed in a cadaver porcine aorta, the histomorphologic appearance of the material was interpreted as being consistent with that observed in the porcine brain sections (Figure 6).

\section{PHYSICAL EXAMINATION}

Despite the embolisation of the hydrophilic coating, none of the affected animals exhibited abnormal clinical signs indicative of central neurologic impairment (e.g., mentation, craniofacial deficits, proprioceptive deficits, and ataxia) during the conduct of the study and had no remarkable findings upon physical examination by the institutional lab animal veterinarian at their respective terminal time points. 


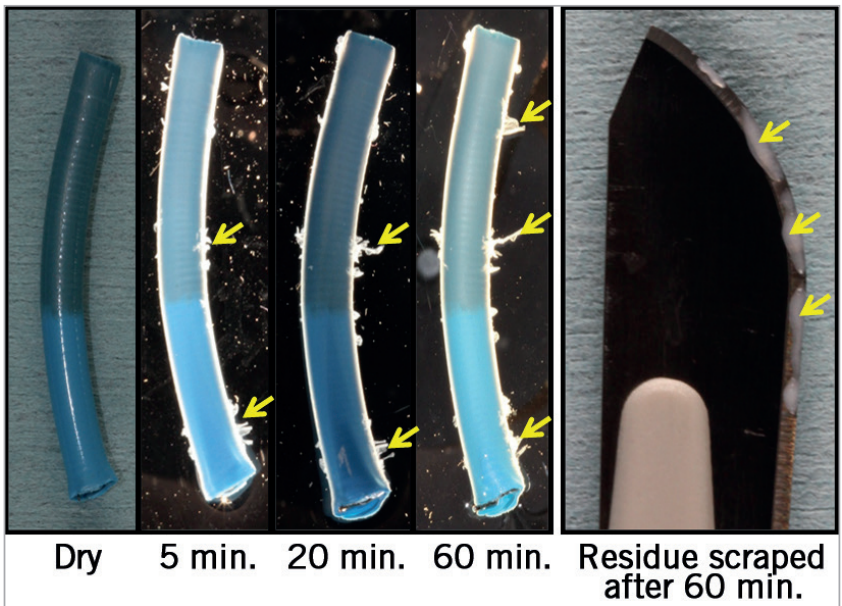

Figure 5. Hydrophilic coating separation. In vitro shedding of the hydrophilic coating from the tip of a 9 Fr guiding sheath placed in $0.9 \%$ saline for 60 minutes.

\section{Discussion}

To our knowledge, this is the first animal study to demonstrate the presence of embolic foreign material in the brain following the use of a hydrophilic material-coated guiding sheath. While the overall purpose of the present studies was to examine the safety of test therapeutic devices, the use of the accessory guiding sheaths in a controlled and consistent environment, with preset durations and follow-up times, allowed us to make relevant in vivo observations of the potential clinically negative impact of these devices and to correlate them with in vitro experiments.

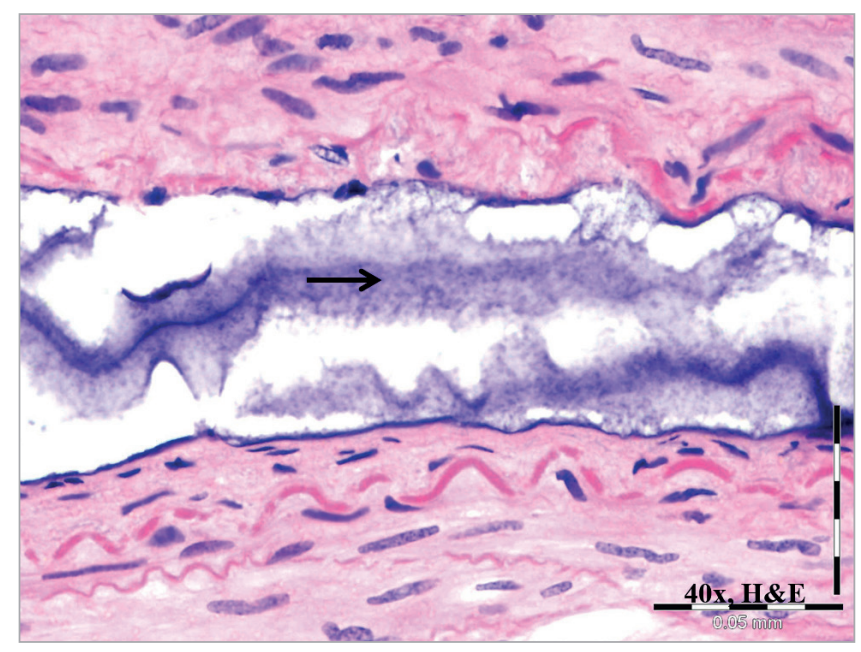

Figure 6. In vitro coating-derived foreign material. Representative image of material removed from surface of a $9 \mathrm{Fr}$ hydrophilic-coated guiding sheath following in vitro submersion in saline as presented in Figure 4 and then placed in a porcine cadaver aorta and processed for histological analysis. Note the amorphous, stippled basophilic material and its resemblance to material shown in Figure 1 and Figure 2.
The observations from the porcine histologic brain sections and the in vitro assessments strongly suggest that the non-biologic material observed in the porcine brains originated from the guiding sheath - in particular, shed and embolised pieces of its hydrophilic PVP coating $\left(\mathrm{AQ}^{\circledR}\right)$ as previously described ${ }^{5}$. Foreign body emboli observed in the control animal in which only the guiding sheath was interventionally deployed (i.e., the animal was never exposed to the test or control delivery systems/stents) appeared morphologically and tinctorially consistent with emboli that were observed in stented animals. Separated/shed material obtained in vitro directly from the surface of a representative segment of the guiding sheath appeared morphologically and tinctorially consistent with that observed in study animals (stented or unstented) and reported in clinical and preclinical publications ${ }^{4,5,7,8}$. In addition, the potential of emboli originating from the SDS is generally precluded as these lacked any coating and were largely shielded from blood flow en route to the stenting site (e.g., iliac arteries). Together, these observations indicate that the source of the foreign material emboli was the guiding sheath. The trend towards an increased incidence of foreign material found among animals accessed via the carotid versus the femoral artery was not statistically significant $(p=0.122)$, and could simply reflect the larger number of animals accessed via carotid only $(n=18)$ versus femoral only $(n=3$, two other animals were accessed by both femoral and carotid). However, based on the guiding sheath's propensity to shed at the vascular access site as indicated in the radial artery inflammation literature, it could reflect the potential for more antegrade shedding of material to the brain following carotid access which would not be possible with femoral access.

Despite a relatively low per plane incidence of foreign material in the histologic brain sections of test animals, the overall incidence of foreign material in test animals over the 180-day study duration was high (52\%). The low per plane incidence in the histologic brain sections of test animals was interpreted as reflecting the low number of sections examined per test brain $(n=6 /$ brain $)$, as increased regional and histologic sampling of the brain, as performed in the one unstented control animal ( $n=28 /$ brain), was associated with an increase in foreign material incidence. The generally benign inflammatory response observed with the foreign material in these animals, the rare incidence of significant adverse effects (e.g., infarction) and the clinically silent nature are consistent with what has been previously reported ${ }^{5}$.

A review of the literature has provided many examples of hydrophilic material-coated catheters associated with percutaneous intervention-associated adverse events. Most numerous are clinical reports of post-intervention radial artery inflammation unassociated with bacterial infection ${ }^{1,2,9-12}$. Adverse event reports obtained from a search of the FDA Manufacturer and User Facility Device Experience (MAUDE) database demonstrated 174 cases of radial artery inflammation over a period of 10 years $^{2}$. All were associated with the same or similar type of PVP-coated Cook sheaths examined herein. Hydrophilic coating material has also been identified histologically in heart ${ }^{7,8,13,14}$, lung ${ }^{4,15}$, brain ${ }^{1,3-5}$, kidney ${ }^{8}$, 
AV graft-associated ${ }^{8}$, and foot ${ }^{4}$ following percutaneous access with a catheter having a hydrophilic coating. The histomorphologic and tinctorial appearance of the embolic material reported for the hydrophilic PVP coating in the literature both ex $v i v o^{1,3,4,7}$ and in patient autopsy or biopsy samples ${ }^{1,3,8,13,14}$ was consistent with that observed in the brain sections of stented or unstented swine in the current study (Figure 1-Figure 4). The reporting of fewer adverse events with hydrophilic-coated catheters which do not employ PVP $P^{4,11,16}$ suggests that the risk for coating embolisation can be minimised through the use of less abrasive coatings, and highlights the urgent need for further evaluation of this class of device in preclinical animal and benchtop models.

\section{STUDY LIMITATIONS}

Caution is warranted in translating our findings to humans, as the arteries in young, healthy swine may be different from the arteries of patients with respect to mechano-anatomic properties which could potentiate catheter abrasion and risk for coating embolisation (e.g., calcification, compliance, length and tortuosity). In addition, although the cerebral blood supply of humans is generally interpreted as comparable to that of swine ${ }^{17}$, some differences exist, most notably a vascular meshwork/plexus (i.e., rete mirabile) situated between the carotid arteries and cerebral vessels of swine ${ }^{18}$, which could serve to filter hydrophilic coating emboli, resulting in a lesser amount of cerebral emboli in swine compared to humans ${ }^{19}$. Nevertheless, the incidence of embolic material observed in our swine model, despite the absence of vascular disease and the presence of an intervening vascular meshwork, suggests prudent observation in comparable clinical scenarios.

\section{CLINICAL IMPLICATIONS}

Hydrophilic coatings add to the ease of sheath use and are increasingly used in sheath technology. However, there appears to be a price to pay - these materials are not as adherent as more hydrophobic material and can avulse in vitro and embolise in animal models to critical organs such as the brain. In the present study, the greatest incidence of foreign material was observed with hydrophilic guiding sheaths involved in carotid arterial access. Though generally avoided in the past, carotid access is resurging with carotid stenting ${ }^{20,21}$ and percutaneous aortic valve placement ${ }^{22-24}$. The lack of sequelae in the physically examined pigs, even in the face of a high incidence of emboli in the cerebellum, may indicate a legitimate lack of effect from the reserve capacity of the porcine brain to withstand embolic insult, and marked anatomic differences between pigs and human cerebral vasculature. The cerebral circulation in the pig is far more developed and anastomotic than in the human, which may reduce not only the incidence of particles reaching the brain but also their distribution patterns within different brain regions. The concern for marked hypersensitivity in a small subset of hypersensitive individuals further amplifies the potential risks associated with our animal findings. Validation and scrutiny in clinical settings is therefore warranted, especially for neuromuscular and cognitive dysfunction.

\section{Conclusions}

The histomorphologic similarity of the foreign material found in the porcine brains to material taken from a sample guiding sheath (and to foreign material reported in the literature) indicates that this material originated from the hydrophilic coating on the guiding sheaths used in the present study. Distribution to the brain in experimental animals has not previously been reported but raises another potential clinical concern around the use of this device, thus warranting further documentation of effect and monitoring in clinical scenarios.

\section{Impact on daily practice}

Hydrophilically coated guidewires, sheaths and catheters facilitate the treatment of otherwise challenging anatomies, yet increase the risk for intravascular shedding and embolisation to the brain and heart in a manner that has been underappreciated. Interventionists are advised to consider the relative risk for particulate shedding from accessory endovascular devices as part of treatment planning, and to monitor patients for neurological symptoms up to several weeks post intervention.

\section{Funding}

E. Edelman is supported in part by a grant from the NIH (R01 GM 49039). CBSET, Inc. provided full support for the work of K. Regan, A. LaRochelle, G. Wong, P. Markham, L. Bailey, A. Spognardi, J. Stanley, A. Tzafriri, B. Zani and G. Kopia.

\section{Conflict of interest statement}

The authors have no conflicts of interest to declare.

\section{References}

1. Fealey ME, Edwards WD, Giannini C, Piepgras DG, Cloft H, Rihal CS. Complications of endovascular polymers associated with vascular introducer sheaths and metallic coils in 3 patients, with literature review. Am J Surg Pathol. 2008;32:1310-6.

2. Zellner C, Ports TA, Yeghiazarians Y, Boyle AJ. Sterile radial artery granuloma after transradial procedures: a unique and avoidable complication. Catheter Cardiovasc Interv. 2010;76:673-6.

3. Barnwell SL, D'Agostino AN, Shapiro SL, Nesbit GM, Kellogg JX. Foreign bodies in small arteries after use of an infusion microcatheter. AJNR Am J Neuroradiol. 1997;18:1886-9.

4. Mehta RI, Mehta RI, Solis OE, Jahan R, Salamon N, Tobis JM, Yong WH, Vinters HV, Fishbein MC. Hydrophilic polymer emboli: an under-recognized iatrogenic cause of ischemia and infarct. Mod Pathol. 2010;23:921-30.

5. Hu YC, Deshmukh VR, Albuquerque FC, Fiorella D, Nixon RR, Heck DV, Barnwell SL, McDougall CG. Histopathological assessment of fatal ipsilateral intraparenchymal hemorrhages after the treatment of supraclinoid aneurysms with the Pipeline Embolization Device. J Neurosurg. 2014;120:365-74.

6. National Research Council. Guide for the Care and Use of Laboratory Animals. National Academy Press, Washington, DC, 1996. 
7. Babcock DE, Hergenrother RW, Craig DA, Kolodgie FD, Virmani R. In vivo distribution of particulate matter from coated angioplasty balloon catheters. Biomaterials. 2013;34:3196-205.

8. Sequeira A, Parimoo N, Wilson J, Traylor J, Bonsib S, Abreo K. Polymer embolization from minimally invasive interventions. Am J Kidney Dis. 2013;61:984-7.

9. Kozak M, Adams DR, Ioffreda MD, Nickolaus MJ, Seery TJ, Chambers CE, Ettinger SM, McNulty PH, Gilchrist IC. Sterile inflammation associated with transradial catheterization and hydrophilic sheaths. Catheter Cardiovasc Interv. 2003;59:207-13.

10. Subramanian R, White CJ, Sternbergh WC, Ferguson DL, Gilchrist IC. Nonhealing wound resulting from a foreign-body reaction to a radial arterial sheath. Catheter Cardiovasc Interv. 2003;59:205-6.

11. Blasco A, Oteo JF, Fontanilla T, Salamanca J, Ocaranza R, Goicolea J. Unusual complications of cardiac catheterization via the radial artery. Rev Esp Cardiol. 2005;58:1233-5.

12. Ziakas A, Karkavelas G, Mochlas S. Sterile inflammation after transradial catheterization using a hydrophilic sheath: a case report. Int J Cardiol. 2005;99:495-6.

13. El-Najjar V, Robinson M. Autopsy demonstration of intramyocardial polymer gel emboli associated with a giant-cell reaction following cardiac catheterization: a case report. Cardiovasc Pathol. 2012;21:59-61.

14. Schipper ME, Stella PR, de Jonge N, Virmani R, de Weger RA, Vink A. Embolization of hydrophilic coating material to small intracardial arteries after multiple percutaneous transluminal angioplasty procedures. Int J Cardiol. 2012;155:e45-6.

15. Allan RW, Alnuaimat H, Edwards WD, Tazelaar HD. Embolization of hydrophilic catheter coating to the lungs: report of a case mimicking granulomatous vasculitis. Am J Clin Pathol. 2009; 132:794-7.

16. Swaminathan RV, Wong SC. Radial access site inflammatory reaction to a recently available hydrophilic coated sheath. Catheter Cardiovasc Interv. 2011;77:1050-3.

17. Ashwini CA, Shubha R, Jayanthi KS. Comparative anatomy of the circle of Willis in man, cow, sheep, goat, and pig. Neuroanatomy. 2008; 7:54-65.

18. Burbridge B, Matte G, Remedios A. Complex intracranial arterial anatomy in swine is unsuitable for cerebral infarction projects. Can Assoc Radiol J. 2004;55:326-9.

19. Karasawa J, Touho H, Ohnishi H, Kawaguchi M. Rete mirabile in humans--case report. Neurol Med Chir (Tokyo). 1997;37:188-92.

20. Guimaraens L, Theron J, Casasco A, Cuellar H. Carotid artery stenting by direct percutaneous puncture. J Vasc Surg. 2011;54: 249-51.

21. O'Steen M, Dougherty K, Strickman NE. Percutaneous cervical carotid artery access with stenting of the left internal carotid artery in an elderly patient. J Geriatr Cardiol. 2007;4:111-4.

22. Guyton RA, Block PC, Thourani VH, Lerakis S, Babaliaros V. Carotid artery access for transcatheter aortic valve replacement. Catheter Cardiovasc Interv. 2013;82:E583-6.

23. Daly MJ , Blair PH, Modine T, Donnelly PM, Jeganathan R, Manoharan G, Spence MS. Carotid-Access Transcatheter Aortic Valve Replacement in a Patient with a Previous Mitral Valve Replacement. J Card Surg. 2014 Mar 10. [Epub ahead of print].

24. Modine T, Lemesle G, Azzaoui R, Sudre A. Aortic valve implantation with the CoreValve ReValving System via left carotid artery access: first case report. J Thorac Cardiovasc Surg. 2010; 140:928-9. 